\title{
Performance Evaluation of Turbo Codes in High Speed Downlink Packet Access Using EXIT Charts
}

\author{
Abiodun Sholiyi ${ }^{1}$, Tim O'Farrell ${ }^{2}$, Omar A. Alzubi ${ }^{3}$ and ${ }^{*}$ Jafar A. Alzubi ${ }^{4}$ \\ ${ }^{1}$ Intel Corporation, Santa Clara California, USA \\ ${ }^{2}$ The University of Sheffield, Sheffield, UK \\ ${ }^{3,4}$ Al-Balqa Applied University, Al-Salt, Jordan \\ 1'Abi.Sholiyi@intel.com,2t.ofarrell@sheffield.ac.uk, ${ }^{3}$ o.zubi@bau.edu.jo, \\ j.zubi@bau.edu.jo
}

\begin{abstract}
This paper investigates the Bit Error Rate (BER) performance of modern channel codecs in Additive White Gaussian Noise (AWGN) and wireless systems. In addition, it explores the characterization of the High Speed Downlink Packet Access (HSDPA) capacity using the EXtrinsic Information Transfer (EXIT) chart technique at different code rates over different modulation schemes.

Furthermore, the EXIT charts of Turbo Code were plotted at different code rates and modulation schemes showing their distance to capacity in bits per channel use. The BER performance of the Turbo Codes is studied with a view of examining how close they are to capacity. Distance to capacity is measured in two ways. First, is the energy per bit to noise power spectral density ratio $\left(E_{b} / N_{0}\right)$ distance to capacity and the other is the distance in bits per channel use.
\end{abstract}

Keywords: Information Theory, Iterative Decoding, EXIT Charts, High Speed Downlink Packet Access.

\section{Introduction}

Since the introduction of channel capacity by Shannon, the distance of channel codecs to capacity has been used to determine their efficiency [1]. A tool that can be used to determine the capacity of a channel and the corresponding capacity loss of a channel codec in bits per channel use is the EXIT chart. The EXIT chart is also a tool used to predict the converging $E_{b} / N_{0}$ values of iterative decoding schemes [2] [3]. To predict if an iterative decoding scheme will converge (i.e. give negligible BER) at a particular $\mathrm{E}_{\mathrm{b}} / \mathrm{N}_{0}$, an EXIT chart is plotted at that $\mathrm{E}_{\mathrm{b}} / \mathrm{N}_{0}$ value. An open tunnel gap in the EXIT chart suggests convergence, while closed or crossed-over tunnel gaps suggest no convergence [4]. In an EXIT chart, the narrower the tunnel gap, the closer the iterative decoding scheme is to capacity [5]. This is the key procedure used in designing and improving iterative decoding schemes. The EXIT chart, which is an acronym for extrinsic information transfer chart developed by S. Ten Brink [4] [6], is a graphical description of the convergence process for iterative decoding. In EXIT charts, the transfer characteristics based on mutual information is used to describe the flow of extrinsic information through the soft input soft output constituent decoders of an iterative decoder [7]. It is a tool used for a clearer understanding of the convergence behavior of iterative decoding schemes. A decoding trajectory is then used to visualize the exchange of extrinsic information between the constituent decoders. The EXIT chart is a tool used to find the least $\mathrm{E}_{\mathrm{b}} / \mathrm{N}_{0}$ to keep the tunnel gap in the EXIT chart open as well as an alternative means to compute the capacity of a channel [8]. 


\section{Related Work}

Recently, the convergence behavior of iterative decoding was investigated after the discovery of Turbo Codes by Berrou et. al. in [9]. Richardson et. al. in [10] proposed a density evolution algorithm to calculate convergence thresholds for low-density parity check codes (LDPC) in an AWGN channel. Density evolution is a message passing algorithm used in predicting iterative decoding such as the LDPC code by analyzing the distribution of message exchange during decoding. The prediction performs well in very long codes. This is so because, as the codeword length tends to infinity, the codebook will be more and more likely to be cycle free, thereby ensuring that the input messages of each node during decoding are independent and could be calculated iteratively. Another group of authors in [11] and [12] studied the convergence of iterative decoders based on Signal to Noise Ratio (SNR) measures, while Peleg et. al. [13], examined a combination of SNR measures and mutual information for the description of inner rate one codes. A novel method for visualizing the convergence behavior of iterative decoding schemes was then proposed by Stephan [6] in his electronics letter titled "Convergence of iterative decoding" where each constituent decoder was represented by a mutual information transfer characteristic which described the flow of extrinsic information through the soft input-soft output (SISO) decoder. The exchange of extrinsic information between constituent decoders is plotted in an EXtrinsic Information Transfer (EXIT) chart. The new concept - at that time - was then illustrated for an iterative demapping and decoding scheme [6]. Stephan did not claim to present a rigorous proof of stability and convergence of iterative decoding, however, his simulation results suggested that the EXIT chart accurately predicted the convergence behavior of the iterative decoding for large interleaver depths.

There are several reasons for choosing EXIT charts as found in [14]:

- Mutual information seems to be the most accurate statistics in predicting the likelihood of decoders in correcting noise corrupted bits [7,15], depending on the SNR value used.

- Mutual information is the most robust statistic, i.e., they accurately predict decoding performances in any channel condition and modulation scheme used. For instance, EXIT functions apply to erasure channels without change. They further apply to symbol-based decoders [16] and to suboptimal decoders such as hard-decision decoders.

In his work, Stephan went on to describe and predict the iterative convergence behavior for both parallel [17] and serially concatenated codes [18] with the use of an EXIT chart. EXIT chart has since then been widely used for the prediction and design of iterative decoding schemes by various authors as seen in the work of Michael Tuchler [19] design of serially concatenated systems for long or short block length. In [19] EXIT chart was used to construct simple irregular codes, which can significantly improve the convergence behavior of iterative decoding.

In a thought-provoking paper, Eiko Seidel et. al. [2], studied two different parallel concatenated coding schemes by way of EXIT chart prediction. One of the schemes was the classical Turbo Code (TC) [7], while the other was the concatenation of regular lowdensity parity-check codes (LDPC). A simplified simulation was set up to obtain the EXIT characteristics for the constituent encoders and applied to parallel concatenated codes for both LDPC and TC. Predictions to know if the LDPC and the TC would converge were then made with the use of an EXIT chart in iterative decoding, showing good performance of the parallel concatenated code (PCC) schemes [2] at particular SNR values. From their chart, it was possible to see that the Turbo Code showed a better performance than the concatenated regular LDPC codes in terms of cliff Energy per bit to Noise power spectral density ratio $\left(\mathrm{E}_{\mathrm{b}} / \mathrm{N}_{0}\right)$, which is usually the dominant criterion for selection of coding schemes in communication systems. 
Eiko Seidel et. al. also showed in [2] that LDPC parallel concatenated code on the other hand converges faster to low bit error rates once the cliff $\mathrm{E}_{\mathrm{b}} / \mathrm{N}_{0}$ is exceeded.

Tee et. al. [20] in their paper entitled " EXIT-chart aided near-capacity irregular bitinterleaved coded modulation design" were able to exploit the use of EXIT chart in the design of a joint inner-outer EXIT matching algorithm in order to design an Irregular Convolution Code (IrCC). Irregular Unitary Rate Code (IrURC) and Irregular Mapper (IrMapper) arrangement for obtaining a narrow but still open EXIT tunnel, which indicated a near-capacity operation as it will be explained later in this paper. The works of Maunder in [21] fully exploits the use of an EXIT chart in designing a near-capacity operation for a joint source channel coding.

\section{Turbo Code in High Speed Downlink Packet Access (HSDPA)}

In. many of today's wireless systems, the Turbo Code is the standard channel codec in use for reliable information transmission. Examples of these are CDMA2000, Universal Mobile Telecommunication System (UMTS i.e. 3G cellular standard), the HSDPA (3.5G cellular standard) and the Long Term Evolution (LTE). Also by puncturing or inserting bits, Turbo Codes can be designed to operate at any code rate with its natural rates as $1 / 2$, $1 / 3,2 / 3$ and $3 / 4$.

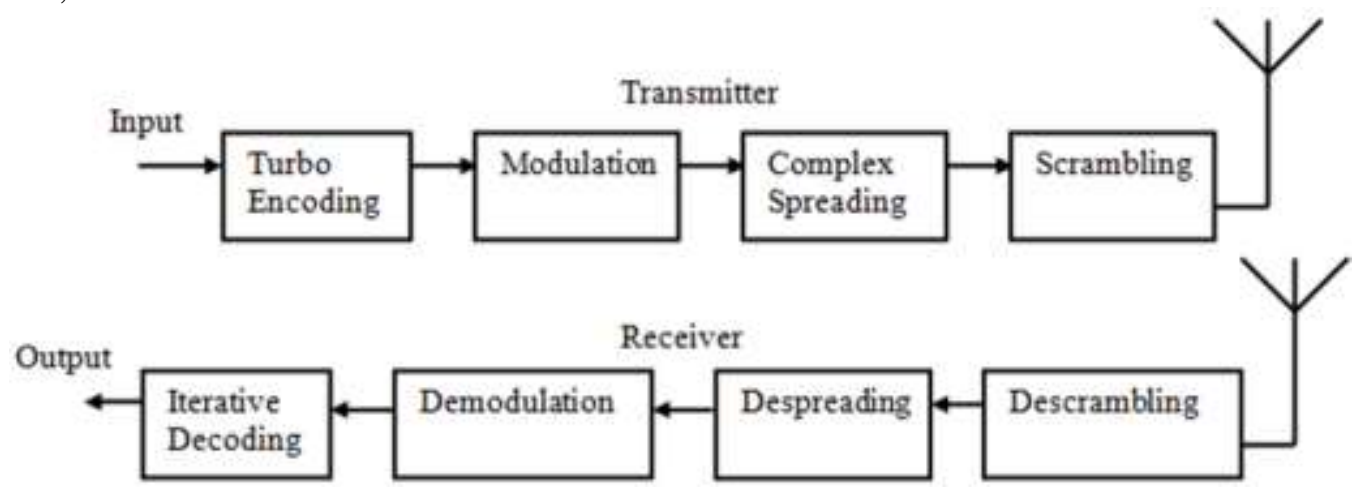

Figure 1. Schematic of HSDPA System Model

The development of the Turbo Code in [9] brought about a new concept in channel coding, which is the exchange of extrinsic information between decoders. This obviously seems to be the strength of a Turbo Code which is also the source of the name "Turbo Code". Today's HSDPA system uses UMTS Turbo Code as depicted in figure 1. The 3GPP W-CDMA system as described in the standard [22], but adapted for the HSDPA was simulated in this paper. At the transmitter, a binary stream is segmented into blocks encoded with a UMTS Turbo Code having octal 13 as the feedback and octal 15 as the feed forward taps. The symbols are then modulated and spread by Walsh codes with a spreading factor of 16 , by assigning one modulation symbol per Walsh code. The spread signals are then multiplied with the complex scrambling code at the same chip rate, before transmitting over the I and Q channels, so that components from the I and Q channels cross over. The scrambling codes are essentially segments of long Gold codes, combined to create complex sequences, as described in [22].

In order to return the symbols to their normal order at the receiver, the complex conjugate of the same scrambling code is applied. Normal order here refers to descrambling and restoring the data bits into the originally spread signal for despreading. The despreader does the exact opposite of the complex spreading using the original spreading factor to despread the descrambled signal. After descrambling, despreading and demodulation, the received bit symbols are passed into an iterative decoder for decoding. This scenario is for a Gaussian channel which does not require a channel interleaver. 
The Convolutional Turbo Code (CTC) consists of two Convolutional codes in parallel, with the original data fed as input to the upper Convolutional code, while the lower Convolutional code receives an interleaved version of the original data. These Convolutional codes could be either recursive systematic Convolutional codes or nonsystematic Convolutional codes.

The recursive systematic Convolutional (RSC) code is usually preferred for use in a Turbo Code to the non-systematic Convolutional (NSC) code basically because in the latter, the input bits do not appear at its output. This simply means the codeword contains merged data and parity bits and cannot be separated into distinct regions of data and parity bits as desired in a Turbo Code. Hence, the RSC ensures that there is a clear distinction between the input data bits and the parity bits at the output of the encoder.

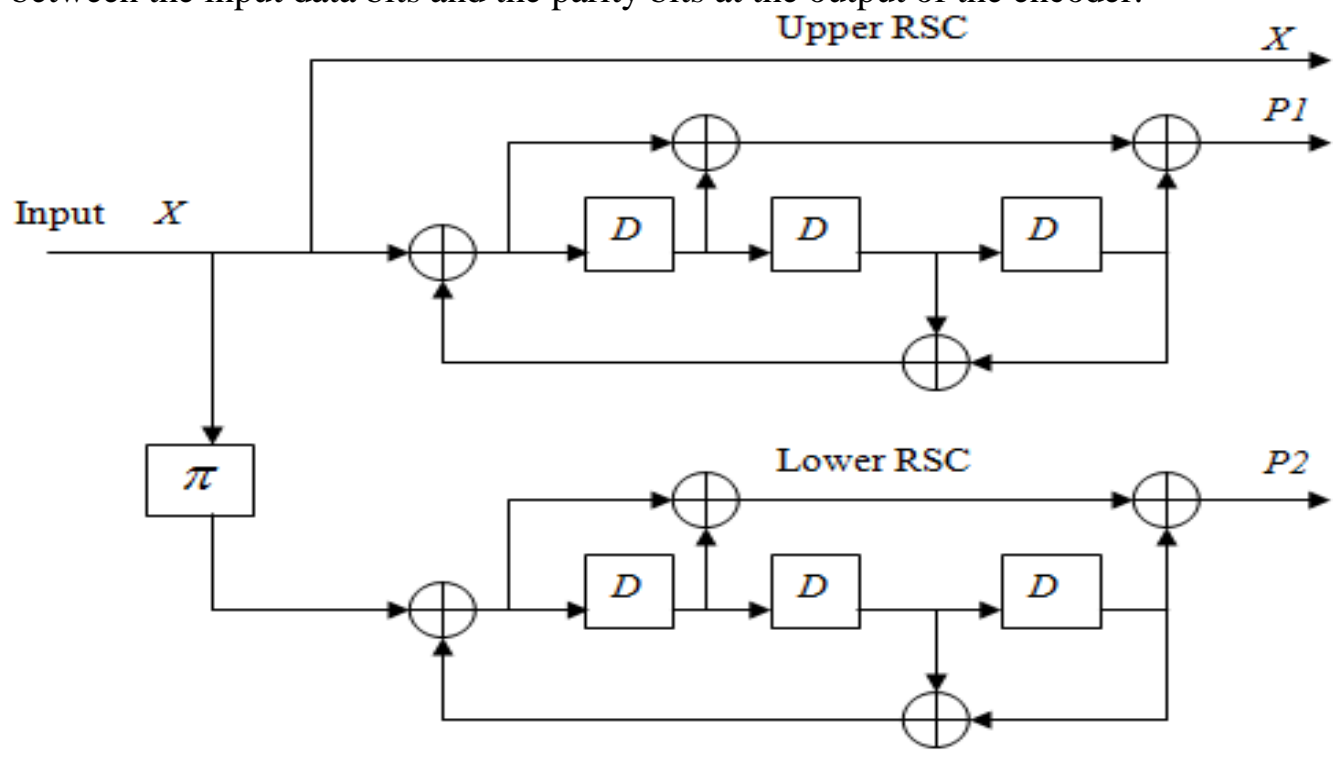

Figure 2. The UMTS Turbo Code Encoder Structure

Another key feature of the RSC is the feedback line from its output to the input. This feedback line in RSCs is to increase the minimum weight of code words which becomes dependent on the interleaver depth when used to construct Turbo Codes. This is not so for an NSC. Hence the RSC has been used in the construction of Turbo Codes such as the UMTS Turbo Code which is used in this paper.

The octal representation of UMTS generator is shown in figure 2 with a feedback and feed forward polynomial of 13 and 15, respectively, for the RSCs. In figure 2, X represents the information bit; P1 and P2 represent the punctured parity bits from the upper and lower RSCs respectively. The interleaver represented by $\pi$, while D represents the memory registers. Modulo 2 additions is used as the numeric system for the CTC.

The Log-MAP version of the BCJR (forward, backward decoding algorithm) [23] has been used as the soft-input soft-output which uses the log likelihood ratios of the received channel information to correct as many noise corrupted bits as possible.

UMTS Turbo Code rates used were $1 / 2$ and $1 / 3$. The simulations were performed using BPSK, QPSK, 16QAM and 64QAM modulation schemes in the AWGN channel. The $\log$ MAP version of the BCJR algorithm was used as the decoder in all cases. For each simulation, a curve showing the BER versus $E_{b} / N_{0}$ is graphed, where the noise energy is the single-sided noise spectral density $N_{0}$ of the channel. 


\section{Bit Error Rate Performance of Turbo Code in Different Modulation Schemes}

In this section, the BER performance of CTC is evaluated via simulations in four modulation schemes at code rates $1 / 2$ and $1 / 3$ to determine their distances to channel capacity in terms of $E_{b} / N_{o}$ value. In the next section, the distance to capacity in bits per channel use was also evaluated. To determine their distance to capacity in bits per channel use, the EXIT charts of the CTCs were plotted and the area properties of their respective EXIT charts were then used to determine the capacity of the channel in bits per channel use.

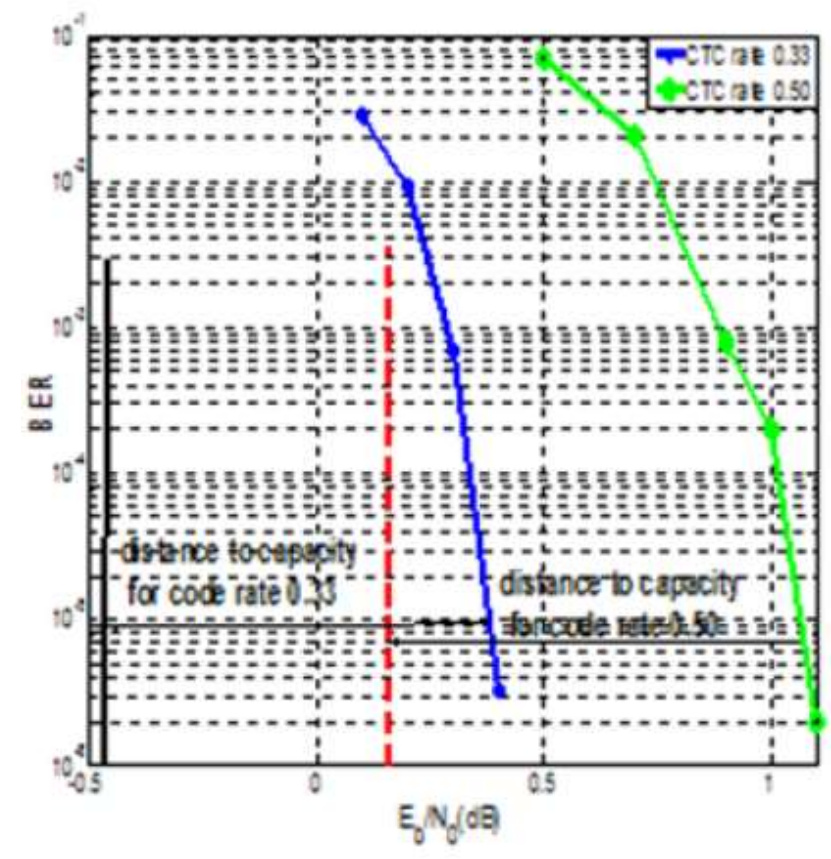

Figure 3. BER Performance of CTC in BPSK

The evaluation was examined in an AWN channel. The evaluation also takes into account the number of iterations required to achieve a very low bit error rate in the region of $10^{-5}$. Random bits of zeros and ones were generated and encoded with the CTC.

The encoded bits were then sent through the channels named above. The received corrupted bits were then sent into the turbo decoder, where the ratio of the number of bits in error to the total number of randomly generated bits was computed. Gray mapping was used for the QPSK, 16QAM and 64QAM modulation schemes. Figures 3 to 6 illustrate the BER versus $\mathrm{E}_{\mathrm{b}} / \mathrm{N}_{0}$ performance of a CTC as used in an HSDPA system model.

The BER curve results for the WCDMA (UMTS) 3GPP Turbo Code in [24] are the same as the BER curves shown in this section. The BER curves shown in figures 3 to 6 have the characteristic of a waterfall region, which is typical of a Turbo Code as the probability of error falls sharply as the $\mathrm{E}_{\mathrm{b}} / \mathrm{N}_{0}$ increases. 


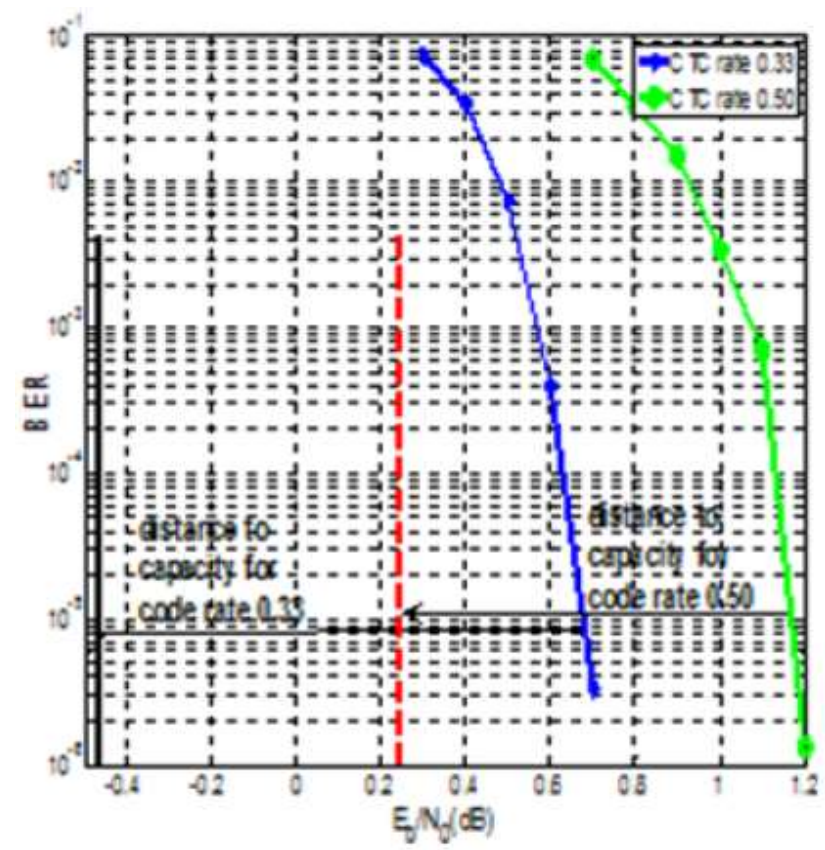

Figure 4. BER Performance of CTC in QPSK

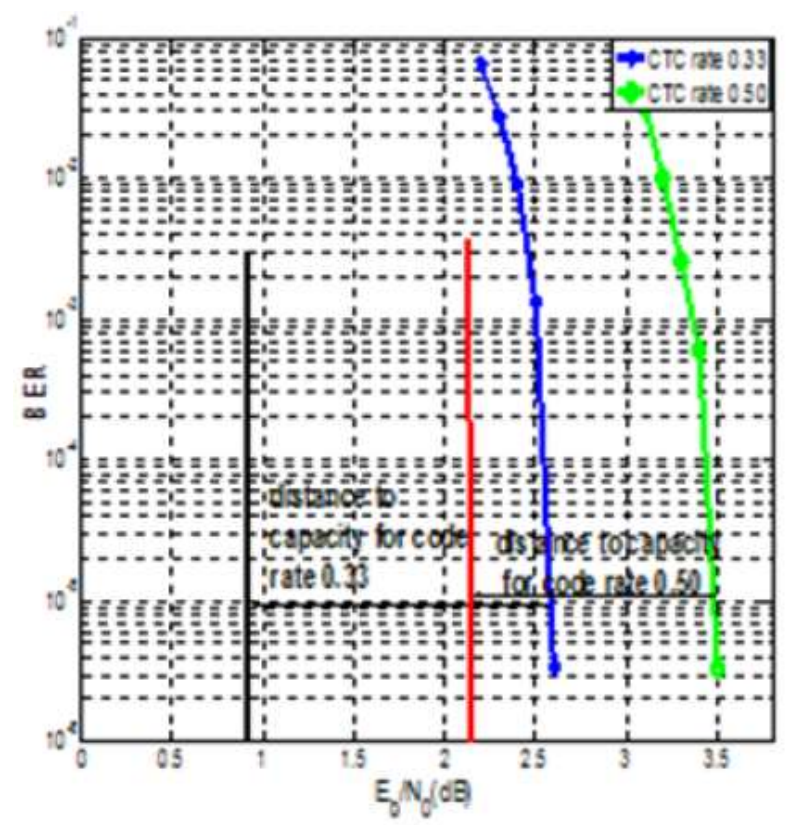

Figure 5. Figure 5. BER Performance of CTC in 16GAM 


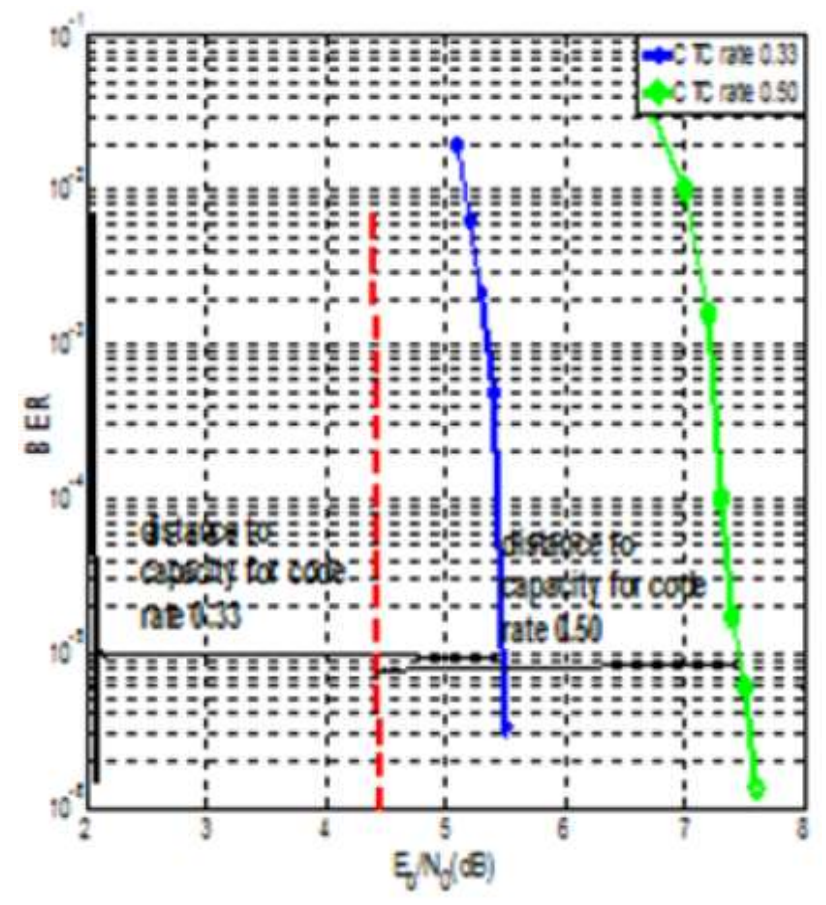

Figure 6. BER Performance of CTC in 64GAM

This characteristic of a Turbo Code makes it one of the reasons it has been used as a channel codec in modern communication systems besides its closeness to the Shannon bound as illustrated in figures 3 to 6 .

Table 1 shows the performance of the CTC tabulated together with their corresponding throughput per channel use which is given by:

$S=R \times\left(\log _{2} M\right)(1-F E R)$

Table 1. CTC Converging $E_{b} / N_{0}$ Values

\begin{tabular}{|c|c|c|c|c|c|}
\hline $\begin{array}{c}\text { Modulation } \\
\text { Scheme }\end{array}$ & Code rate & $E_{B} / E_{0}(d b)$ & $S$ & $\begin{array}{c}\text { Number of } \\
\text { iterations }\end{array}$ & $\begin{array}{c}\text { Shannon's capacity } \\
E_{B} / E_{0} \\
(d b)\end{array}$ \\
\hline BPSK & $1 / 3$ & 0.400 & 0.329 & 11 & -0.430 \\
\hline BPSK & $1 / 2$ & 1.100 & 0.490 & 15 & 0.210 \\
\hline QPSK & $1 / 3$ & 0.700 & 0.665 & 15 & -0.41 \\
\hline QPSK & $1 / 2$ & 1.180 & 0.997 & 11 & 0.230 \\
\hline 16QAM & $1 / 3$ & 2.600 & 1.328 & 14 & 0.750 \\
\hline 16QAM & $1 / 2$ & 3.500 & 1.993 & 21 & 2.300 \\
\hline 64QAM & $1 / 3$ & 5.500 & 1.996 & 24 & 2.300 \\
\hline 64QAM & $1 / 2$ & 7.500 & 2.960 & 19 & 4.500 \\
\hline
\end{tabular}

$S$ stands for the throughput value in bits per channel use.

Where $\mathrm{R}$ is the rate of the code, FER is the frame error rate and $\mathrm{M}$ is the M-ary order of the modulation scheme. The frame error rate is computed by dividing the number of frames in error after decoding by the total number of frames used in the simulation. In determining the FER the number of frames corresponding to at least ten frames in errors per $E_{b} / N_{0}$ value was used. A frame in error means, a single or more bits in the frame is received in error after decoding. Table 1 also shows converging $E_{b} / N_{0}$ values for the 
CTCs at different code rates and modulation schemes. It can be deduced from table 1 that the higher the modulation order, the further their distances to Shannon's capacity.

Shannon's capacity in this paper is measured first in $\mathrm{dB}$, which is the minimum $\mathrm{E}_{\mathrm{b}} / \mathrm{N}_{0}$ required to achieve a low BER for a Discrete-input Continuous-output Memoryless Channel (DCMC), and is a function of the modulation scheme and code rate [1]. Secondly, capacity is measured by the maximum possible throughput in bits per channel use at a particular $\mathrm{E}_{\mathrm{b}} / \mathrm{N}_{0}$ value at which information can be sent with arbitrarily low probability of error [24], (i.e. the maximum mutual information per single channel use) which is also a function of the modulation scheme and code rate denoted as CDCMC in this paper. However, CDCMC as a measure of distance to capacity takes into account the system's spectral efficiency as well as the energy efficiency unlike the $E_{b} / N_{0}$ in $d B$ which only takes into account the energy efficiency, making CDCMC a better option.

The performance of the CTC in a 64QAM modulation scheme is worse than other modulation schemes in terms of throughput value against required $\mathrm{E}_{\mathrm{b}} / \mathrm{N}_{0}$ to achieve a BER of $10^{-5}$.

This is mainly due to the dense constellation cluster (together with the number of bits per symbol) required for a 64QAM modulation scheme, thereby requiring a far higher $\mathrm{E}_{\mathrm{b}} / \mathrm{N}_{0}$ value to achieve a very low probability of error. As an illustration, a CTC in a 64QAM modulation scheme at a code rate of $1 / 3$ would give a throughput value of about 1.996 bits per channel use at an $\mathrm{E}_{\mathrm{b}} / \mathrm{N}_{0}$ value of $5.4 \mathrm{~dB}$. In comparison to the CTC in a 16QAM modulation scheme, a bit per channel use of 1.993 requires an $\mathrm{E}_{\mathrm{b}} / \mathrm{N}_{0}$ value of 3.4 $\mathrm{dB}$ which is a significant $2 \mathrm{~dB}$ less than that required for the CTC in a 64QAM scheme for the same throughput. In this section, the BER performance of the CTC in a Gaussian channel with four different modulation schemes was examined, with a view to determining their distances to their respective $\mathrm{E}_{\mathrm{b}} / \mathrm{N}_{0}$ capacity in $\mathrm{dB}$.

\section{EXIT Charts of Turbo Codes and Their Area Properties}

In this section, we will investigate the extrinsic transfer characteristics using iterative decoding for both parallel and serial concatenation schemes. In addition, we will present the parameters which affect the transfer characteristic curve of an EXIT chart.

\subsection{Extrinsic Transfer Characteristics using Iterative Decoder for Parallel Concatenated Scheme}

The iterative decoder for a Parallel Concatenated Code (PCC), e.g. a Convolutional Turbo Code, is shown in figure 7. By definition from figure 7, $\mathrm{Y}, \mathrm{P}_{1}$ and $\mathrm{P}_{2}$, are the received channel observations corresponding to the information bits, parity bits for the upper decoder and parity bits for the lower decoder, respectively. $Z_{1}$, is composed of the received information bits $\mathrm{Y}$ and its corresponding parity $\mathrm{P}_{1}$, which goes into the upper decoder, while $\mathrm{Z}_{2}$, composed of the received information bit $\mathrm{Y}$ and its corresponding parity $\mathrm{P}_{2}$, goes into the lower decoder. $\mathrm{A}_{1}, \mathrm{~A}_{2}$ are a priori inputs to the upper and lower decoder respectively, with $\mathrm{E}_{1}$ and $\mathrm{E}_{2}$ as part of the a posteriori outputs called extrinsic information, gleaned from the upper and lower decoders respectively. $\mathrm{D}_{2}$ represents the final decoded soft output with $\mathrm{D}_{1}$ a part of the a posteriori output from the upper decoder. The variables $Z_{1}, A_{1}, D_{1}, E_{1}, Z_{2}, A_{2}, D_{2}$, and $E_{2}$ all denote Log-Likelihood Ratios (LLRvalues). 


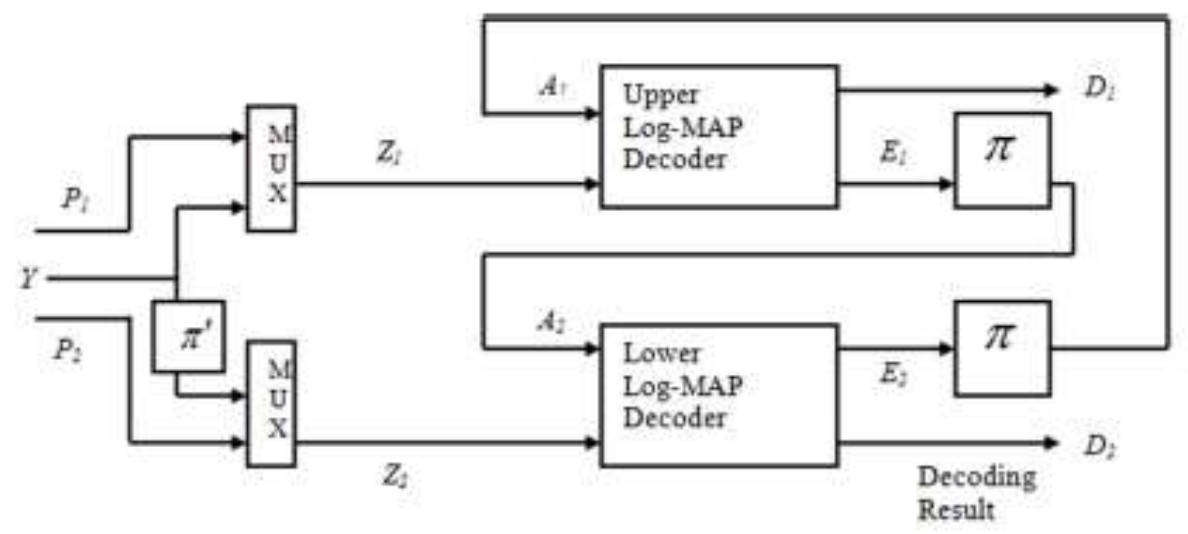

Figure 7. Schematic of Turbo Code Decoding Structure

The upper decoder takes the channel observation $Z_{1}$ on the received systematic (information) bits $\mathrm{Y}$ and respective parity $\mathrm{P}_{1}$ together with an initial a priori input $\mathrm{A}_{1}\left(\mathrm{~A}_{1}\right.$ is zero for the first iteration ) obtained from the lower decoder. The upper decoder then outputs an extrinsic information $E_{1}$ gleaned from itself $\left(E_{1}=D_{1}-A_{1}-Z_{1}\right)$ which is then passed through an interleaver to become the a priori input $\mathrm{A} 2$ of the lower decoder.

The lower decoder then takes the interleaved channel observations $Z_{2}$ on the systematic bits $\mathrm{Y}$ and respective parity bits $\mathrm{P}_{2}$ together with the a priori $\mathrm{A}_{2}$ and gleans an extrinsic information $\left(E_{2}=D_{2}-A_{2}-Z_{2}\right)$, which is fed back into the upper decoder via an interleaver becoming the a priori knowledge $\mathrm{A}_{1}$ for the upper decoder.

A plot of the mutual information (Ia) between the a priori A and transmitted bits $\mathrm{X}$ on the abscissa and the mutual information (Ie) between the extrinsic information $\mathrm{E}$ and transmitted bits $\mathrm{X}$ of the upper decoder on the ordinate, gives the EXIT function of that decoder. A second plot of an "inverted" EXIT function for the lower decoder is used to explain the convergence of the iterative decoding process.

The term "inverted" here simply connotes swapping of the plot's axis, i.e. a priori on the ordinate with the extrinsic information on the abscissa. The mutual information $\mathrm{I}(\mathrm{X}$, A) between the a priori input and transmitted bits $X$ is given as Ia, while the mutual information $\mathrm{I}(\mathrm{X} ; \mathrm{E})$ between the extrinsic output of a decoder and the transmitted bit $\mathrm{X}$ is denoted by Ie. In a typical EXIT chart, $\mathrm{Ia}_{1}$ denotes the mutual information $\mathrm{I}\left(\mathrm{X}, \mathrm{A}_{1}\right)$ between the a priori input to the upper decoder and the transmitted bits $\mathrm{X}, \mathrm{Ie}_{1}$ for the mutual information $\mathrm{I}\left(\mathrm{X} ; \mathrm{E}_{1}\right)$ between the extrinsic output of the upper decoder and the transmitted bits $\mathrm{X}$ with, $\mathrm{Ia}_{2}$ and $\mathrm{Ie}_{2}$ representing the same for the lower decoder respectively. $\mathrm{Ia}_{1}, \mathrm{Ie}_{1}, \mathrm{Ia}_{2}$ with $\mathrm{Ie}_{2}$ range from 0 to 1 in value. It is important to note that interleaving does not change the inherent mutual information meaning $\mathrm{Ia}_{1}$ is actually $\mathrm{Ie}_{2}$ upon de-interleaving as shown in [7]. It should also be noted that, the two EXIT functions in a PCC depends on the $\mathrm{E}_{\mathrm{b}} / \mathrm{N}_{0}$ value being used, simply because the two decoders have inputs from the same channel observation as shown in [14] [3].

\subsection{Extrinsic Transfer Characteristics Using Iterative Decoder for a Serially Concatenated Scheme}

In this section, a detailed explanation of the EXIT chart of Serially Concatenated Codes (SCC) is explained. In SCC, the outermost encoder is known as the outer encoder, while the other is called the inner encoder. The corresponding decoder to each of these encoders is also known as the outer and the inner decoders.

The EXIT chart of a serially concatenated scheme is similar to that of a parallel concatenated scheme except that, the inner code's EXIT function depends on the $E_{b} / N_{0}$, because it directly receives the channel observations, while the outer code's EXIT function does not depend on the $E_{b} / N_{0}$ as it does not directly receive any channel 
observations. Mathematically, to measure the information contents of an a priori knowledge $A$, the mutual information $\mathrm{Ia}=\mathrm{I}(\mathrm{X}, \mathrm{A})$ between the transmitted systematic bits $\mathrm{X}$ and the LLR values of $\mathrm{A}$ as found in [7] are used and given by:

$I_{a}=\frac{1}{2} \cdot \sum_{x=-1,1} \int_{-\infty}^{+\infty} p_{a}(\xi \mid X=x) \times \log _{2}\left[\frac{2 \cdot p_{a}(\xi \mid X=x)}{p_{a}(\xi \mid X=-1)+p_{a}(\xi \mid X=+1)}\right] d \xi$

With $p_{a}(\xi \mid X=x)$ representing the conditional probability density function belonging to the LLR A. The mutual information $\mathrm{Ie}=(\mathrm{X}, \mathrm{E})$ between the transmitted systematic bits $\mathrm{X}$ and the LLR values of $\mathrm{E}$ (the extrinsic information) as found in [7] is given by:

$$
I_{e}=\frac{1}{2} \cdot \sum_{x=-1,1-\infty}^{+\infty} p_{e}(\xi \mid X=x) \times \log _{2}\left[\frac{2 \cdot p_{e}(\xi \mid X=x)}{p_{e}(\xi \mid X=-1)+p_{e}(\xi \mid X=+1)}\right] d \xi
$$

With $p_{e}(\xi \mid X=x)$ representing the conditional probability density function belongs to the LLR E. The mutual information for an LLR frame at a particular value (say 0, 0.1, $0.2, \ldots \ldots, 1.0)$ depends on the distribution of the LLR values in that frame corresponding to zero-valued bits and that of the LLR values in the frame pertaining to unity-valued bits. If the distribution of the LLR values that corresponds to zero-valued bits equals that of the LLR values pertaining to unity-valued bits, then the mutual information will be zero. In this case, the LLR values are totally unreliable and the selection of a bit's logical value based upon the sign of the corresponding LLR will only give the correct answer $50 \%$ of the time [21].

To Compute the mutual information in transmission systems with a posteriori (APP) decoders, the "averaging method is capable of calculating the mutual information of an LLR frame without considering the corresponding originally transmitted bit frame X, provided that the LLR is generated by an optimal APP decoder. Another method used in calculating mutual information is the histogram method [7] [21]. In this paper, the histogram and the averaging methods were used and found to have very similar results.

This illustrates the fact that employing either method would yield a correct EXIT chart. The results generated in this paper were from the histogram method. One key factor in generating an EXIT Chart is that $\mathrm{Ie}_{1}=\mathrm{Ia}_{2}$ must be well interleaved with large interleaver depth to ensure that they are uncorrelated. To plot an EXIT Chart, all that is required is the mutual information between these LLR's i.e. $I(X, A)$ and $I(X, E)$ with $I(X, A)=H(X)$ $-\mathrm{H}(\mathrm{X} / \mathrm{A})$ and $\mathrm{I}(\mathrm{X}, \mathrm{E})=\mathrm{H}(\mathrm{X})-\mathrm{H}(\mathrm{X} / \mathrm{E})$.

\subsection{Parameters Influencing the Transfer Characteristic Curve of an EXIT Chart}

There are some important parameters that impact on the transfer characteristic curve of EXIT charts. These are:

- Constraint length of the code in the case of a Convolutional code.

- Depth of interleaving.

- Different code polynomials.

- $\mathrm{E}_{\mathrm{b}} / \mathrm{N}_{0}$.

- Shape of the inverted EXIT function.

EXIT charts for the UMTS CTCs in an HSDPA model at their converging $\mathrm{E}_{\mathrm{b}} / \mathrm{N}_{0}$ values for various code rates and modulation schemes are shown by way of example in figures 8(a) to 8(i). 


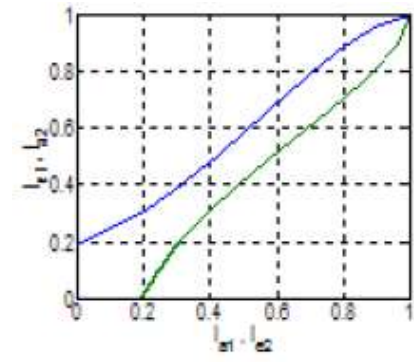

(a) $0.4 \mathrm{~dB}, \mathrm{BPSK}$, rate $\frac{1}{3}$

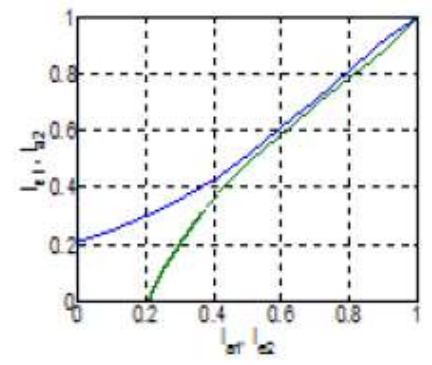

(d) $0.7 \mathrm{~dB}, \mathrm{QPSK}$, rate $\frac{1}{3}$

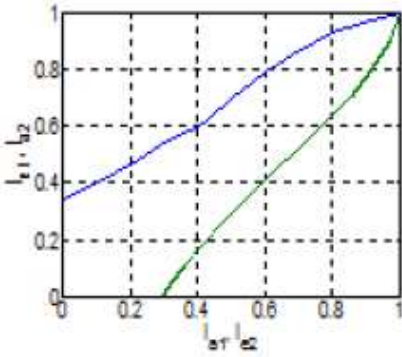

(g) $3.5 \mathrm{~dB}, 16 \mathrm{QAM}$, rate $\frac{1}{2}$

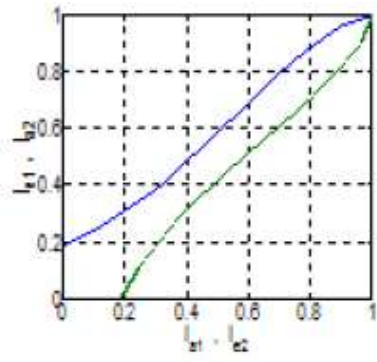

(b) $1.1 \mathrm{~dB}, \mathrm{BPSK}$, rate $\frac{1}{2}$

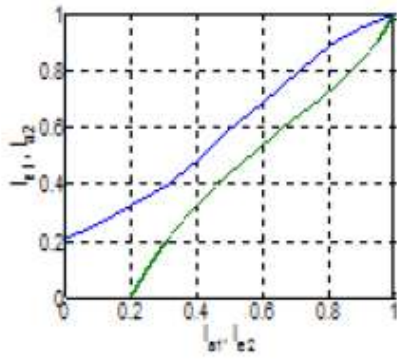

(e) $1.18 \mathrm{~dB}, \mathrm{QPSK}$, rate $\frac{1}{2}$

(f) $2.6 \mathrm{~dB}, 16 \mathrm{QAM}$, rate $\frac{1}{3}$

Figure 8. EXIT Charts for CTC at code rate $1 / 3$ and 1/2. Legend: ' $E_{b} / N_{0}$ value', 'modulation code rate'

Many of the EXIT charts shown in figure 8 have an open tunnel gap until the point (1, 1). The open tunnel gap denotes that a very low bit error rate during bit transmission at those $\mathrm{E}_{\mathrm{b}} / \mathrm{N}_{0}$ values will occur. When the gap closes or crosses over before the $(1,1)$ point, the BER does not converge to a negligible value (i.e. the case of figure 8(c)). The scenario in figure $8(d)$ is the same as that of figure $8(\mathrm{c})$ but with a higher $\mathrm{E}_{\mathrm{b}} / \mathrm{N}_{0}(0.1 \mathrm{~dB}$ difference $)$ giving an open but very narrow tunnel gap. A Parallel concatenated code has two decoders in parallel, which can be called, upper and lower decoders. In the case of a serially concatenated code, the two decoders are serially concatenated and can be called inner and outer decoders. 
Table 2. EXIT Chart and Area Properties of UMTS Convolutional Turbo Code

\begin{tabular}{|c|c|c|c|c|c|c|c|}
\hline $\begin{array}{c}\text { Modulation } \\
\text { Scheme }\end{array}$ & Code rate & $E_{B} / E_{0}(d b)$ & $S$ & $C_{D C M C}$ & $C_{A}$ & $L$ & $\% \Delta$ \\
\hline BPSK & $1 / 3$ & 0.400 & 0.329 & 0.394 & 0.392 & 0.065 & 16.497 \\
\hline BPSK & $1 / 2$ & 1.000 & 0.490 & 0.570 & 0.5810 .08014 .035 \\
\hline QPSK & $1 / 3$ & 0.700 & 0.665 & 0.820 & 0.731 & 0.155 & 18.902 \\
\hline QPSK & $1 / 2$ & 1.100 & 0.997 & 1.157 & 1.1560 .16013 .829 \\
\hline 16QAM & $1 / 3$ & 2.500 & 1.328 & 1.700 & 1.2970 .372 & 21.882 \\
\hline 16QAM & $1 / 2$ & 3.400 & 1.993 & 2.313 & 2.675 & 0.32013 .834 \\
\hline 64QAM & $1 / 3$ & 5.400 & 1.996 & 2.840 & 2.6050 .844 & 29.718 \\
\hline 64QAM & $1 / 2$ & 7.500 & 2.960 & 3.900 & 3.507 & 0.94024 .103 \\
\hline
\end{tabular}

$S, C_{D C M C}, C_{A}$, and $L$ in the table stand for the throughput value, Discrete-input Continuous-output Memoryless Channel capacity, attainable capacity, and capacity loss, respectively. The unit of all these variables is bits per channel use. $\% \Delta$ is the percentage loss in capacity with respect to $C_{D C M C}$.

In [25], the authors have shown that the area $\mathrm{A}_{\text {in }}$ underneath the EXIT function of an inner code for a serially concatenated code can be given by:

$A_{\text {in }}=\int_{0}^{1} I_{e}\left(I_{a}\right) d I_{a}=\frac{I(X, Y)}{R_{i n}}$

Where $I_{e}$ is the extrinsic output values for the inner decoder, $I_{a}$ the a priori input values for the inner decoder, $\mathrm{I}_{\mathrm{e}}$ is a function of $\mathrm{I}_{\mathrm{a}}$, i.e. $\mathrm{I}_{\mathrm{e}}\left(\mathrm{I}_{\mathrm{a}}\right), \mathrm{I}(\mathrm{X}, \mathrm{Y})$ is the maximum mutual information transfer between the transmitted symbol $\mathrm{X}$ and received symbol $\mathrm{Y}$, also known as capacity, and $R_{i n}$ is the rate of the inner code. $I_{e}$ which is a function of $I_{a}$ is integrated with respect to $I_{a}\left(i . e . d_{a}\right.$ ).

This implies that for a rate one inner code, the area underneath the inner code equals the capacity of the communication channel and for inner codes with $\mathrm{R}_{\text {in }}<1$, the area underneath the inner code is an attainable capacity (a slightly lower capacity bound). For a given modulation scheme, this attainable capacity $\mathrm{C}_{\mathrm{A}}$ can be calculated by:

$C_{A}=A_{\text {in }} \times R_{\text {in }} \times \log _{2} M$

Where, $\mathrm{M}$ is the M-ary order of the modulation scheme. In the case of the parallel concatenated code, the sum of areas underneath the EXIT functions for the CTCs equals the attainable capacity $C_{A}[14]$ given by:

$A=\left(A_{u}+A_{l}\right)=\int_{0}^{1} I_{e}\left(I_{a}\right) d I_{a}=\frac{I(X, Y)}{R}$

Where $\mathrm{Au}$ is the area underneath the EXIT function of the upper code, $\mathrm{A}_{\mathrm{I}}$ as the area underneath the EXIT function of the lower code and $\mathrm{R}$ is the code rate of the parallel concatenated scheme. Table 2 shows the use of the area properties of the EXIT chart in the calculation of attainable capacity for a PCC (i.e. a CTC) at their converging $E_{b} / N_{0}$ values. In table 2 , the various attainable capacities have been used to compute the distance of a parallel concatenated code such as the CTC to capacity in bits per channel use at their converging $\mathrm{E}_{\mathrm{b}} / \mathrm{N}_{0}$ values. This computation was made in four different modulation 
schemes. CDCMC in table 2 is the Discrete-input Continuous-output Memoryless Channel capacity, while $\mathrm{L}$ is the capacity loss in bits per channel use (i.e. CDCMC - S). The attainable capacity for the BPSK and 16QAM code rate $1 / 2$ in table 2 is slightly larger than the DCMC capacity. This is due to some inconsistency in the EXIT charts of short frame size as explained in [5].

Table 2 shows the distance from capacity in bits per channel use for each modulation and code rate for a CTC in HSDPA for an AWGN channel. Table 2 shows that the 64QAM modulation scheme (code rate $1 / 3$ and $1 / 2$ ) is the furthest from the capacity bound in bits per channel use.

\section{Conclusions}

In this paper, the bit error rate performance of the convolutional turbo code is considered in a Gaussian noisy channel. More specifically, the CTC's BER performance was evaluated using an HSDPA system model with a Gaussian channel. These BER performances were evaluated in four different modulation schemes; BPSK, QPSK, 16QAM and 64QAM. Additionally, a characterization of the HSDPA capacity using the EXIT chart technique was given.

It was seen in this paper, that the EXIT charts of the CTCs at their converging $\mathrm{E}_{\mathrm{b}} / \mathrm{N}_{0}$ values all had open tunnel gaps. Furthermore, it can be deduced that the CTCs code rate $1 / 3$ were further away from capacity in comparison with the CTC code rate $1 / 2$ with the same modulation scheme percentage wise. Clearly, these results make the code rate $1 / 2$ CTC a better choice in terms of spectral efficiency. It was also established in this paper that, the sum of areas underneath the EXIT functions of the upper and lower decoders of a CTC sums up to the capacity of the channel.

\section{References}

[1] A. Sholiyi, J. A. Alzubi, O. A. Alzubi, O. Almomani, and T. O'Farrell, "Near capacity irregular turbo code," Indian Journal of Science and Technology, vol. 8, no. 23, (2015).

[2] A. G. E. von Elbwart, J. Lohr, and E. Seidel, "Performance comparison of parallel concatenated codes using EXIT chart prediction", Proceedings of the IEEE 15th International Symposium on Personal, Indoor and Mobile Radio Communications, PIMRC, (2004); Barcelona, Spain.

[3] A. Sholiyi, B. Badic, R. Joyce, and T. O?Farrell, "A characterization of hsdpa capacity using EXIT chart techniques," Proceedings of the IEEE NGAMAST, (2009); Cardiff, Wales.

[4] J. Hagenauer, "The exit chart - introduction to extrinsic information transfer", Iterative Processing,? Proc. 12th Europ. Signal Proc. Conf (2004), pp. 1541-1548.

[5] J. Lee, and R. E. Blahut, "Generalized exit chart and BER analysis of finite-length turbo codes," in Proceedings of IEEE Global Telecommunications Conference, GLOBECOM, (2003), pp. 2067-2072.

[6] S. T. Brink, "Convergence of iterative decoding," Electronics Letters, vol. 35, no. 10, (1999), pp. 806808.

[7] S. T. Brink, "Convergence behavior of iteratively decoded parallel concatenated codes." IEEE Trans. Communications, vol. 49, no. 10, (2001), pp. 1727-1737.

[8] M. Tuchler and J. Hagenauer, "Exit charts of irregular codes", (2002).

[9] C. Berrou, A. Glavieux, and P. Thitimajshima, "Near Shannon limit error-correcting coding and decoding: Turbocodes. 1," Communications, 1993. ICC '93 Geneva. Technical Program, IEEE International Conference, vol. 2, (1993), pp. 1064-1070.

[10] T. J. Richardson and R. L. Urbanke, "The capacity of low density parity-check codes under messagepassing decoding," Information Theory, IEEE Transactions, vol. 47, no. 2, (2002), pp. 599-618.

[11] H. El Gamal and A. R. Hammons, "Analyzing the turbo decoder using the Gaussian approximation," IEEE J. Select Areas Comm., vol. 47, (2001), pp. 671-686.

[12] D. Divsalar, S. Dolinar, and P. F., "Low complexity turbo like codes", Proceedings of 2nd Int. Symp. Turbo Codes, (2000), pp. 73-80.

[13] M. Peleg, I. Sason, S. Shamai, and A. Elia, "On interleaved, differentially encoded convolutional codes,” IEEE Trans. Information Theory, vol. 45, no. 7, (1999), pp. 2572-2582.

[14] A. Ashikhmin, G. Kramer, and S. T. Brink, "Extrinsic information transfer functions: Model and erasure channel properties," IEEE Transactions on Information Theory, vol. 50, no. 11, (2004).

[15] M. Tuchler, S. T. Brink, and J. Hagenauer, "Measures for tracing convergence of iterative decoding algorithms", Proc. 4th IEEE/ITG Conf. on Source and Channel Coding, (2002); Berlin, Germany. 
[16] B. Scanavino, G. Montorsi, and S. Benedetto, "Convergence properties of iterative decoders working at bit and symbol level," Proceedings of IEEE Global Telecommunications, Conference ,GLOBECOM, vol. 2, (2001), pp. 1037-1041.

[17] S. T. Brink, "Iterative decoding trajectories of parallel concatenated codes", Proc. 3rd IEEE/ITG Conference on Source and Channel Coding, (2000), pp. 75-80.

[18] O. A. Alzubi, "Performance evaluation of ag block turbo codes over fading channels using pbsk," Proceedings of ACM International Conference on Engineering and MIS, (2015); Istanbul, Turkey.

[19] M. Tuchler, "Design of serially concatenated systems for long or short block length communications," Proceedings of IEEE International Conference ICC '03, vol. 4, (2003), pp. 2948-2952.

[20] R. Y. S. Tee, R. G. Maunder, and L. Hanzo, "Exit chart aided near-capacity irregular bit-interleaved coded modulation design," IEEE Transactions on Wireless Communications, vol. 8, no. 1, (2009), pp. 32-37.

[21] G. Robert and R. G. Maunders, "Irregular variable length coding", PhD Thesis, University of Southampton, U.K, (2007).

[22] S. Abukharis, J. A. Alzubi, O. A. Alzubi, S. Alamri, and T. O'Farrell, "Packet error rate performance of IEEE 802.11g under bluetooth interface", Research Journal of Applied Sciences, Engineering and Technology, vol. 8, no. 12, (2014), pp. 1419-1423.

[23] L. Bahl, J. Cocke, F. Jelinek, and J. Raviv, "Optimal decoding of linear codes for minimizing symbol error rate (corresp.)," IEEE Trans. Inf. Theory., vol. 20, no. 2, (2006), pp. 284-287.

[24] J. Alzubi, O. Alzubi, and T. M. Chen, "Forward Error Correction Based On Algebraic-Geometric Theory", Springer Publishing Company, Incorporated, (2014).

[25] A. Ashikhmin, G. Kramer, and S. T. Brink, "Code rate and the area under extrinsic information transfer curves", Proceedings of the IEEE International Symposium on Information Theory, (2002); Lausanne, Switzerland. 